\title{
Doing Structural Biology in situ - Prospects and Challenges
}

Juergen M. Plitzko ${ }^{1,2}$, Elizabeth Villa ${ }^{1}$, Ben Engel ${ }^{1}$, Yoshiyuki Fukuda ${ }^{1}$, Tim Laugks ${ }^{1}$, Miroslava Schaffer ${ }^{1}$, Alexander Schwarz ${ }^{1}$, Felix J.B. Bäuerlein ${ }^{1}$, Julia Mahamid ${ }^{1}$, Lena Fitting Kourkoutis ${ }^{3}$, Alexander Rigort ${ }^{1}$ and Wolfgang Baumeister ${ }^{1}$

1. Max Planck Institute of Biochemistry, Dept. Molecular Structural Biology, Martinsried, Germany

2. Utrecht University, Bijvoet Center for Biomolecular Research, Utrecht, The Netherlands

${ }^{3 .}$ Cornell University, Applied and Engineering Physics, Ithaca, NY, USA

One of the ultimate goals in structural biology is the in situ visualization of the concerted action of macromolecular assemblies directly within their cellular context. Cryo-electron tomography (CET) is ideally suited to unveil cellular landscapes at molecular resolution, however only sufficiently small objects $(<500 \mathrm{~nm})$ can be examined in toto with a resolution that is high enough for the detection and identification of molecular structures. For studying eukaryotic cells at molecular resolution it is therefore necessary to develop tools and techniques providing controlled access to structural features buried deep inside cellular volumes [1,2].

Many supramolecular structures of great interest, such as centrosomes, or kinetochores are present only in low copy numbers or are so deeply rooted in their cellular environments that it is not possible to locate them easily or to isolate them without violating their structural integrity. Hence, it is highly desirable to study them in situ.

Moreover, cryo-ET is challenged by the following paradox: Although the information content of a small volume increases (as one zooms further and further into the cell) sampling statistics decrease (as the volume imaged represents only a small proportion of the cell). Therefore approaches are needed that can be applied to a wide range of sample sizes (e.g. cells and tissue), that can bridge several orders of magnitude in spatial resolution and that provide a minimum level of statistical significance. To study biological systems in situ and at different scales (e.g. from 'cells to atoms'), we need methods which are capable of navigating, targeting, examining and analyzing complex samples in their native environment. However, a successful combination of various methodologies into a robust and reliable workflow is a major challenge. Here we present a streamlined workflow that integrates and successfully combines three different methodologies for in situ structural biology on frozen hydrated biological specimens:

(i) correlative cryo-fluorescence microscopy to localize features in all three dimensions within frozenhydrated cells,

(ii) cryo-focused ion beam milling to thin previously identified subcellular structures in a targeted manner (Fig.1), and

(iii) cryo-electron tomography to allow the structural analysis of the cellular environment in situ at macromolecular resolution $(\sim 2 \mathrm{~nm})$.

Explicitly, we aim to discuss approaches to improve the localization specificity and precision in $3 \mathrm{D}$, the preparation reproducibility and yield and furthermore the overall throughput, all together key factors in advancing in situ imaging for structural biology [3].

References:

[1] M Marko et al, Nat Methods 4(3) (2007) p.215

[2] A Rigort et al, PNAS 109/12 (2012) p.4449.

[3] The authors acknowledge funding from the European Commission in the 7th Framework Program (grant agreement HEALTH-F4-2008-201648/PROSPECTS). 

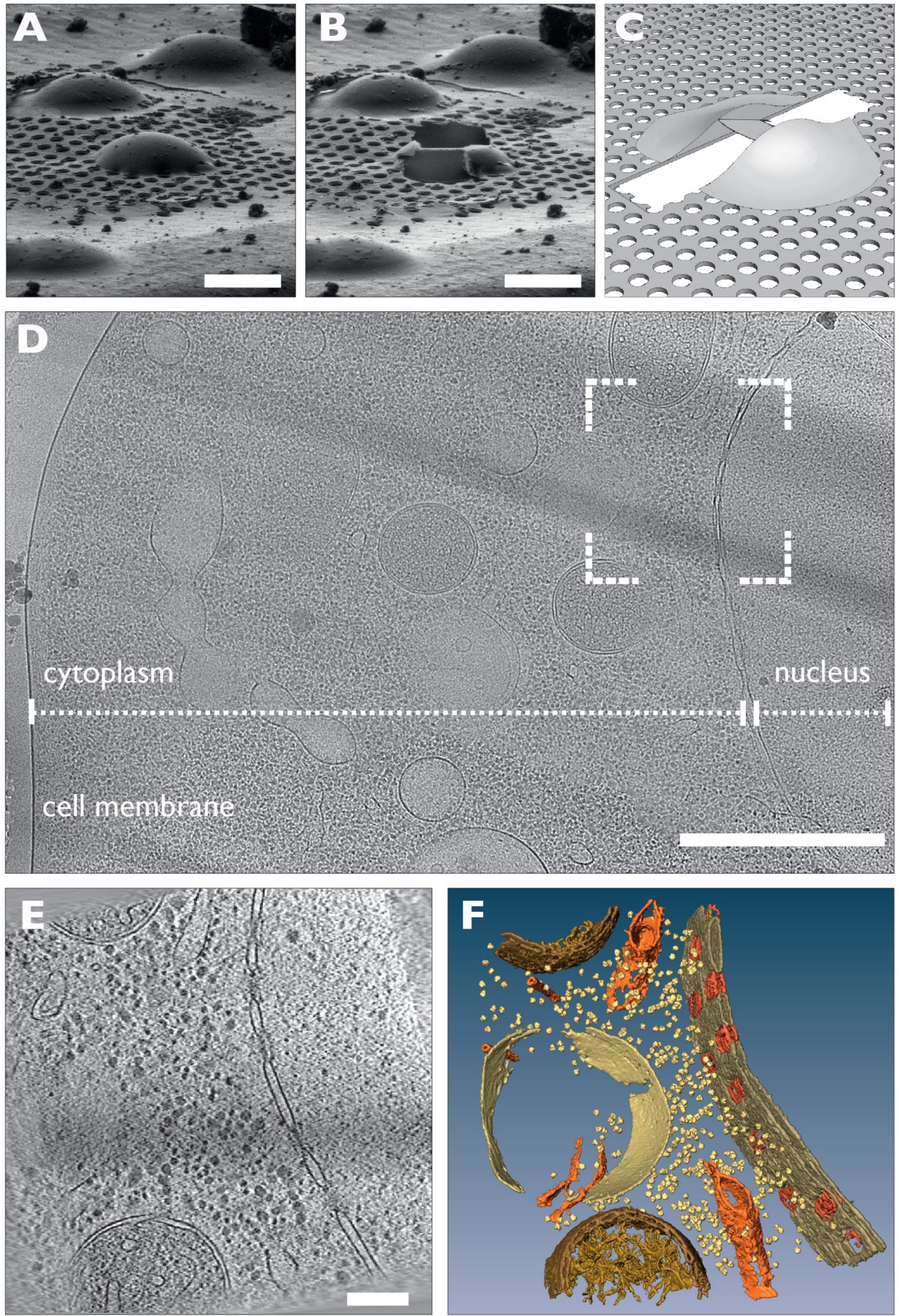

Figure 1: FIB lamella milling of a frozen-hydrated eukaryotic cell (D. discoideum). (A) FIB/SEM micrograph of the cell embedded in a thin layer of ice on the carbon support film. (B) Corresponding region after FIB milling. (C) Schematic. (D) TEM micrograph showing the prepared area, approx. 25 $\mu \mathrm{m}^{2}$. (E) Slice from tomographic reconstruction of the boxed region in (D). (F) Surface rendered visualization of the tomographic volume from (E). Scale bars: (A,B) $5 \mu \mathrm{m}$, (D) $1 \mu \mathrm{m}$, (E) $200 \mathrm{~nm}$. 